\title{
Una aplicación de la teoría del comportamiento planificado al segmento masculino latinoamericano de productos de cuidado personal
}

DOI: https://doi.org/10.21158/01208160.n83.2017.1821
Fecha de recepción: 23 de abril de 2017

Fecha de aprobación: 23 de octubre de 2017

Otto Regalado Pezúa ${ }^{1}$ ESAN Graduate School of Business oregalado@esan.edu.pe

Carlos Alberto Guerrero Medina ${ }^{2}$ ESAN Graduate School of Business cguerrero@esan.edu.pe

Raúl Francisco Montalvo Corzo ${ }^{3}$ EGADE Business School del Tecnológico de Monterrey rmontalvo@itesm.mx

Cómo citar este artículo / To reference this article / Comment citer cet article / Para citar este artigo:

Regalado Pezúa, O., Guerrero Medina, C. A y Montalvo Corzo, R. F. (2017). Una aplicación de la teoría del comportamiento planificado al segmento masculino latinoamericano de productos de cuidado personal. Revista EAN, 83, pp 141- 163. https:// doi.org/10.21158/01208160.n83.2017.1821

\section{Resumen}

Este estudio busca identificar los factores que influyen en el consumo de productos de cuidado personal dentro del segmento masculino latinoamericano. Para ello, se aplicó un instrumento basado en la teoría del comportamiento planificado a 600 hombres pertenecientes a dos países de la región con diferentes niveles de desarrollo de la categoría en cuestión: México - alto desarrollo-, y Perú - moderado desarrollo - . Los datos obtenidos fueron analizados a partir de un modelo de ecuaciones estructurales. Los resultados sugieren, que en ambos países estudiados, la norma subjetiva inhibiría el consumo de este tipo de productos entre los hombres. Así mismo, el impacto de la norma subjetiva sería menor en hombres jóvenes, teniendo ellos mayor apertura al consumo de estos productos.

\section{Palabras clave}

Marketing estratégico, intención de consumo, productos de belleza, cuidado personal, consumidor masculino, comportamiento del consumidor.

\footnotetext{
${ }^{1}$ Doctor en Gestión de las Organizaciones y DEA en Ciencias de gestión por la Université de Nice Sophia Antipolis, Niza. Máster en Marketing Cuantitativo por la Université Pierre Mendes, France, Grenoble. Magíster en Administración por ESAN Graduate School of Business. Licenciado en Ciencias Administrativas por la Universidad de Lima. Investigador, jefe del área académica de marketing y director comercial de ESAN Graduate School of Business.

${ }^{2}$ Candidato al grado de Doctor en Ciencias económicas y empresariales de la Universidad de Granada, España. Máster en Marketing Intelligence por ESIC Business \& Marketing School, España. MBA por ESAN Graduate School of Business. Licenciado en Sociología por la Pontificia Universidad Católica del Perú. Profesor e investigador del área de marketing de ESAN Graduate School of Business.

${ }^{3}$ Doctor en Economía por la University of Essex, Inglaterra. Maestro en Economía por el Centro de Investigación y Docencia Económicas, AC (CIDE) en México. Licenciado en Sistemas de Computación Administrativa. Estudios de estancia en Economía Geográfica y Generaciones Traslapadas en la Universidade Técnica de Lisboa; Negocios y Mercado Chino en Fudan University y Peking University; Visualización de Datos en Stevens Institute of Technology y Emprendimiento en Babson College. Director de la EGADE Business School Guadalajara.
} 


\title{
An application of the behavioral theory in the latinamerican male segment of personal care products
}

\begin{abstract}
This research study identifies those factors which influence the consumption of care products in the Latinamerican male segment. That's why, an instrument based on the behavorial theory was applied to 600 men living in two countries of this region and having different levels of development regarding the above mentioned category, Mexico - highly developed - and Peru - moderately developed-. The data obtained were analyzed using a model of structural equations. The results show that a subjective norm will stop men's consumption of these kinds of products. Similarly, the impact of this subjective norm will be lower in young men, allowing them to have a great aperture in the consumption of these care products.
\end{abstract}

Key words. Strategic marketing, consuming intention, beauty and personal care products, male consumers, consumer behavior.

\section{Application de la théorie du comportement planifié au segment masculin des produits de soin personnel en Amérique latine}

Résumé. Cette étude tente d'identifier les facteurs influant sur la consommation des produits de soin personnel du segment masculin en Amérique latine. Un instrument de mesure, basé sur la théorie du comportement planifié a été utilisé et appliqué à 600 hommes appartenant à deux pays de la région possédant différents niveaux de développement: le Mexique - développement important-et le Pérou - développement modéré-. Les données obtenues ont été analysées à partir d'un modèle d'équations structurelles. Les résultats suggèrent que, dans les deux pays étudiés, la norme subjective inhiberait la consommation de ce type de produits chez les hommes. De la même manière, l'impact de cette norme subjective serait moins fort chez le public jeune plus réceptif à la consommation de ce type de produits.

Mots clefs. Marketing stratégique, intention de consommation, produits de beauté et de soin personnel, consommateur masculin, comportement du consommateur.

\section{Uma aplicação da teoria do comportamento planejado ao consumo de produtos de cuidados pessoais pelo segmento masculino latino-americano}

Resumo. Este estudo procura identificar os fatores que influenciam o consumo de produtos de cuidados pessoais no segmento masculino latino-americano. Para isso, aplicou-se um instrumento baseado na teoria do comportamento planejado a 600 homens pertencentes a dois países da região com diferentes niveis de desenvolvimento da categoria em questão: México - alto desenvolvimento - e Peru - desenvolvimento moderado-. Os dados obtidos foram analisados a partir de um modelo de equações estruturais. Os resultados sugerem que, em ambos os países estudados, a norma subjetiva inibiria o consumo desse tipo de produtos entre os homens. Da mesma forma, o impacto da norma subjetiva seria menor em homens jovens, tendo eles maior abertura para o consumo desses produtos.

Palavras-chave. Marketing estratégico, intenção de consumo, produtos de beleza e de cuidados pessoais, consumidor masculino, comportamento do consumidor. 


\section{Introducción}

T a preocupación por la apariencia física se Lha incrementado en las últimas décadas considerándose parte esencial dentro del proceso de construcción de la identidad del individuo y su posterior realización (Sarpila, 2012, Featherstone, 2007; Turner, 2008; Shilling, 2003; Gill, Henwood y McLean, 2005). Esta situación ha contribuido a impulsar el consumo de productos de belleza y cuidado personal dentro del segmento femenino (Sarpila y Räsänen, 2011), el cual es un público tradicionalmente asociado con dicha categoría (Berg y Teigen, 2008; Räsänen y Wilska, 2007). Al respecto, de acuerdo con Euromonitor (2017), entre 2011 y 2016, la categoría belleza y cuidado personal ha crecido en promedio $4.87 \%$ anual en el mundo, situándose América Latina por encima del promedio con un crecimiento de $8.82 \%$ durante ese mismo periodo.

Por otra parte, la creciente preocupación por la apariencia física también ha promovido el ingreso y la adopción de los productos pertenecientes a la categoría en cuestión dentro del segmento masculino (Souiden y Diagne,
2009; Ruiz de Maya y Grande Esteban, 2006). Frente a nuevos patrones de consumo por parte de los hombres, la oferta ha respondido desarrollando nuevos productos enfocados en este segmento (Tournois, 2014; Mintel, 2013; Nizar y Nizar Souiden, 2009). En la actualidad, la subcategoría cuidado personal masculino representa $10.78 \%$ de las ventas totales de la categoría belleza y cuidado personal. En América Latina, este valor alcanza 17.15 $\%$, siendo la región con mayor participación dentro de la categoría, principalmente por la influencia de Brasil (Euromonitor, 2017).

Pese al atractivo del segmento masculino, en cuanto a su tamaño y capacidad adquisitiva, las cifras muestran que los productos de belleza y cuidado personal continúan concentrándose en el público femenino. Tal como se observa, la participación de los productos para hombres dentro de la categoría belleza y cuidado personal no ha ganado terreno en los últimos diez años. Esta tendencia de carácter estable se repite en diferentes regiones en el mundo (Figura 1).

Figura 1. Participación de los productos orientados al segmento masculino dentro de la categoría belleza y cuidado personal.

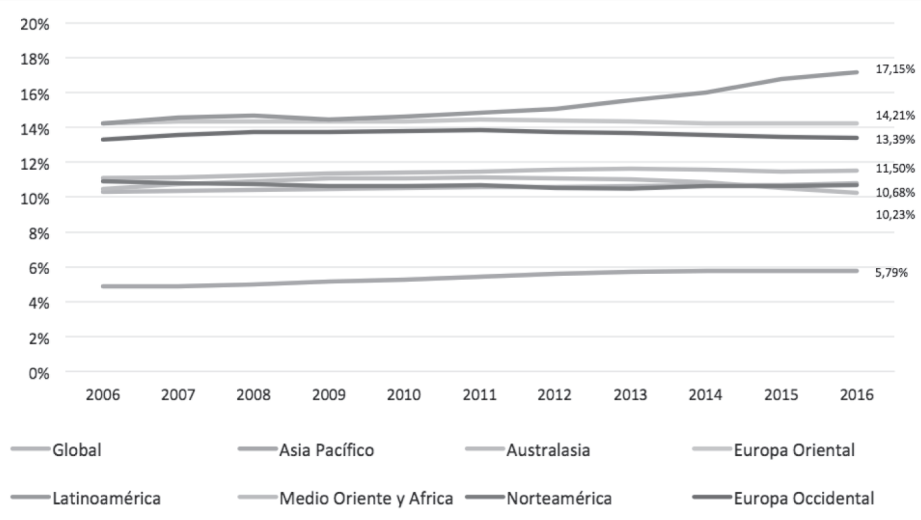

Fuente. Euromonitor, (2017). 
Frente a este contexto, este estudio busca analizar los factores que influyen en el consumo de productos de cuidado personal dentro del segmento masculino en América Latina. Con miras a que los resultados permitan contrastar realidades distintas basadas en el nivel de desarrollo de la categoría belleza y cuidado personal, el estudio aborda dos entornos: uno, donde dicha categoría cuenta con un alto nivel de desarrollo dentro de la región latinoamericana -México-, y otro, cuyo desarrollo aún es moderado -Perú-. Al respecto, en 2016, el mercado de cuidado personal masculino en México alcanzó un valor de USD 1330 millones, siendo superado en la región solo por Brasil, en América Latina, mientras que el mercado peruano tuvo un valor USD 294 millones (Euromonitor, 2017). Los resultados obtenidos servirán de base para el desarrollo de propuestas de valor atractivas dirigidas al público masculino latinoamericano, con miras a dinamizar el consumo de esta categoría de productos.

La investigación está compuesta por seis secciones. La primera de ellas sitúa al lector en el mercado del cuidado personal masculino analizando información relevante vinculada con su evolución en el contexto global y regional. La segunda sección desarrolla el marco teórico. La tercera, presenta el modelo hipotético conceptual sobre el cual se trabaja la investigación. La cuarta, describe la metodología aplicada. La quinta sección presenta el análisis de los resultados realizado. Estos resultados se discuten en la sexta sección, la cual también presenta las conclusiones y limitaciones del estudio.

\section{Marco teórico}

$\mathrm{L}$ a teoría del comportamiento planificado (TPB theory of planned behaviour), propuesta por Ajzen (1991, 2011, 2014), busca predecir una determinada conducta considerando tanto factores internos como externos al individuo, los cuales posibilitan, contextualizan y constriñen la acción. De acuerdo con este modelo, el comportamiento de un individuo es explicado en función de su intención (Kautonen, Van Gelderen y Fink, 2015). Esta última, a su vez, está condicionada por tres factores: i) la actitud positiva o negativa frente a una determinada acción -actitud-; ii) la percepción de aprobación o desaprobación del comportamiento en cuestión por parte de las personas que conforman el entorno del individuo - norma subjetiva-; iii) la percepción acerca de la capacidad para influir en el resultado final basado en los recursos tangibles e intangibles requeridos para llevar a cabo la acción - percepción de control-. Estos tres factores se encuentran relacionados entre sí. La influencia relativa de cada uno de ellos en la intención de actuar, y en la acción misma, varía dependiendo del tipo de conducta que se está estudiando y las situaciones específicas del contexto (Ang, Ramayah y Amin, 2015).

Dentro del terreno del marketing, la TPB ha sido aplicada en diversas investigaciones vinculadas con la forma en que son tomadas las decisiones de compra y consumo de diversos productos y servicios, y comprobar así su pertinencia en diferentes poblaciones, 
conductas y categorías de productos. Por ejemplo, este modelo ha sido utilizado para estudiar la intención de consumo de productos ecológicos (Yadav y Pathak, 2017), la adopción de servicios en línea (Chen y Li, 2010) y los patrones de elección de entidades financieras (Farah, 2017). Por otra parte, investigaciones como las de Jalilvand y Samiei (2012) extendieron los constructos iniciales del modelo con el fin de explicar mejor ciertos fenómenos específicos, como el impacto del e-WOM en la selección de destinos turísticos. De igual forma, Lee, Murphy y Swiller (2009) incluyen como factor adicional el modelo original del hedonismo con miras a explicar la lealtad de los jóvenes hacia los teléfonos móviles.

En el contexto específico de este estudio, la pertinencia de utilizar el modelo de Azjen para explicar el comportamiento de consumo dentro de la categoría cuidado personal masculino se basa en dos premisas. Primero, el modelo ha sido utilizado previamente en productos y patrones de comportamiento vinculados con la apariencia física y el cuidado personal, como el consumo de productos de moda y lujo (Jain, Khan y Mishra, 2017), la realización de actividad física (Wing, Bray y Martin, 2009) y el consumo de alimentos saludables en jóvenes (Fila y Smith, 2006). Más estrechamente con la categoría estudiada, este modelo ha sido empleado como base para explicar el efecto de los valores y la experiencia previa en la intención de compra de productos orgánicos de cuidado de la piel y cabello dentro del segmento femenino (Yeon y Chung, 2011). Segundo, la intención de uso de los productos de cuidado personal es una decisión de carácter individual que no está regida solo por la perspectiva de uno mismo, sino que interviene también el entorno que lo rodea y el contexto que facilita o dificulta el comportamiento (Netemeyer, Burton y
Johnston, 1991). Los factores involucrados en la TPB toman en consideración estos aspectos y permiten una predicción de la intención más allá de la voluntad del propio consumidor y de su control (Leeman y Ong, 2008). Al respecto, investigaciones como las de Sukato y Elsey (2009) han estudiado previamente el comportamiento del consumidor masculino en la compra de productos para el cuidado de la piel tomando como base para su modelo la teoría de la acción razonada (TRA theory of reasoned action) (Ajzen, 1981). Esta propuesta no considera por completo la influencia del contexto que rodea a este comportamiento al obviar como factor la facilidad o dificultad percibida que puede interferir en el control del comportamiento por parte del individuo.

Sobre la base de la TPB, el consumo de productos de cuidado personal por parte del segmento masculino se explicaría a través de la actitud, la norma subjetiva y la percepción de control. A continuación, se detalla cada uno de estos elementos.

La actitud hacia este tipo de productos se refiere a la predisposición a consumirlos. De acuerdo con ello, el individuo realiza un juicio positivo o negativo acerca del comportamiento en cuestión, influenciado por sus valores, experiencias, conocimiento previo y la importancia que para él representa dicha acción (Carpi, Breva y Palermo, 2005; Shih y Fang, 2004; Buttle y Bok, 1996). Esta propuesta guarda relación con la teoría de la decisión de compra familiar de Sheth (1974), según la cual la intención de adquirir un producto de forma autónoma depende de la predisposición que tenga el consumidor hacia este bien. Esta predisposición, a su vez, se desarrolla a partir de las percepciones evaluativas -o creencias previas - acerca del bien en cuestión. De acuerdo con la propuesta de Sheth, la 
actitud o predisposición sería parte esencial del factor cognitivo presente en el proceso de decisión de compra.

La norma subjetiva se refiere a la influencia que tiene el entorno, entiéndase familiares, amigos, personas allegadas y referentes, en el comportamiento de consumo hacia este tipo de productos (Carpi, Breva y Palermo, 2005; Chiou, 1998; Lee y Green, 1991). En este caso, el consumidor toma en cuenta la aceptación o rechazo de determinado grupo para establecer un patrón de comportamiento que se adapte a las expectativas de este. Esto guarda relación con la teoría de la decisión de compra familiar de Sheth (1974), según la cual existe un conjunto de factores que influyen en la intención de adquirir un producto de forma autónoma. Entre estos factores se encuentran las expectativas que el individuo debe cubrir en cuanto a su sexo, rol dentro de la familia, grupo de referencia, clase social, estilo de vida y el ciclo de vida en el que se encuentre su propia familia.

La percepción de control hace referencia a los recursos y las capacidades que considera poseer el consumidor para afrontar trabas externas e internas que le impidan realizar la acción (Lee, Qu y Kim, 2007; Ajzen, 2002). Al igual que en la TRA, este factor no es tomado en consideración de manera explícita por la teoría de la decisión de compra familiar de Sheth (1974), que obvia la relevancia del contexto para favorecer o dificultar la compra.

\section{Modelo conceptual}

$E_{\mathrm{s}}^{\mathrm{s}}$ studios como los de Yeon y Chung (2011), y Sukato y Elsey (2009), dan indicios de la influencia positiva de la actitud hacia los productos de cuidado personal masculino en la intención de compra de estos. La actitud favorable hacia este tipo de productos está asociada con cambios en los intereses de la población masculina, los cuales muestran cada vez mayor preocupación por su imagen personal y atractivo físico (Khan, Dongping, Abdullah, Ahmad, Ahmad Ghauri y Ghazanfar, 2017; Featherstone, 2007; Turner, 2008). Esta mayor preocupación motiva el consumo de productos más especializados dentro de esta categoría (Ruiz de Maya y Grande Esteban, 2006).
En países latinoamericanos como México -alto desarrollo del mercado de productos de cuidado personal masculino-, y Perú - moderado desarrollo del mercado de productos de cuidado personal masculino-, el consumidor muestra interés por bienes y servicios más especializados, debido a la consolidación de la clase media (Euromonitor, 2016; Ipsos Perú, 2013, 2015). Así mismo, la población muestra un distanciamiento de los valores de supervivencia en favor de la adquisición de valores más orientados hacia la autoexpresión (World Values Survey, 2017; Inglehart y Baker, 2000). Esto generaría una actitud de apertura a la prueba de nuevos productos que contribuyan a construir su imagen personal y proyectarla hacia los demás, tales como los pertenecientes a la categoría en cuestión y así favorecer la intención de consumo de estos. 
A partir de ello se desprenden las siguientes hipótesis:

- Hipótesis 1a: en países latinoamericanos con alto desarrollo de la categoría cuidado personal masculino, como el caso de México, la actitud de los hombres por consumir estos productos influiría positivamente en su intención de consumo.

- Hipótesis 1b: en países latinoamericanos con moderado desarrollo de la categoría cuidado personal masculino, como el caso de Perú, la actitud de los hombres por consumir estos productos influiría positivamente en su intención de consumo.

Estudios como los de Souiden y Diagne (2009), Dano, Roux y Nyeck (2003) y Hillhouse, Turrisi y Kastner (2000), dan indicios de la influencia negativa de la norma subjetiva en la intención de compra de productos pertenecientes a la categoría cuidado personal masculino dentro de ciertas sociedades. Souiden y Diagne (2009) sugieren que, en sociedades donde se percibe que estos de productos están orientados a un público femenino, el consumo de estos por parte de los hombres es limitado. Por su parte, Dano, Roux y Nyeck (2003) sugieren que el orden social establece estándares de belleza tanto para hombres como mujeres, y en cada caso empuja a los consumidores a desarrollar una imagen satisfactoria que se encuentre en armonía con lo establecido por su cultura.

En países latinoamericanos como México -alto desarrollo del mercado de productos de cuidado personal masculino-, y Perú - moderado desarrollo del mercado de productos de cuidado personal masculino-, la población cuenta con una fuerte presencia de valores tradicionales en comparación con países como los Estados Unidos, Canadá y las economías del este de Europa (World Values Survey, 2017; Inglehart y Wayne, 2000). Schwartz (1992) define estos valores como el compromiso, el respeto y la aceptación de las costumbres e ideas que una determinada cultura impone a los individuos. Esto implica una mayor presión del entorno y de las convenciones en la toma de decisiones individuales, entre las que se encuentra el consumo.

Frente a la importante presencia de valores tradicionales en países latinoamericanos con alto y moderado desarrollo de la categoría cuidado personal masculino, se esperaría que la norma subjetiva presente una influencia negativa en la intención de consumo de los productos en cuestión. A partir de ello, se desprenden las siguientes hipótesis:

- Hipótesis 2a: en países latinoamericanos con alto desarrollo de la categoría cuidado personal masculino, como en el caso de México, la norma subjetiva de los hombres influiría negativamente en la intención de consumo de estos productos.

- Hipótesis 2b: en países latinoamericanos con moderado desarrollo de la categoría cuidado personal masculino, como en el caso de Perú, la norma subjetiva de los hombres influiría negativamente en la intención de consumo de estos productos.

De acuerdo con Navarro y Delgado (2006), en sociedades donde priman valores tradicionales, los roles sociales y los patrones de consumo se encuentran marcadamente diferenciados por sexo. Conforme las sociedades se alejan de la influencia de los valores tradicionales, estas diferencias en el contexto de roles y patrones se van debilitando. En cuanto a la variable edad, los jóvenes cuentan con menor influencia de los valores tradicionales (World 
Values Survey, 2013), y por ende, los patrones de consumo diferenciados por sexo son más débiles (Mintel, 2017). Al respecto, Coley y Burgess (2003) identificaron que los hombres entre los 16 a 24 años son el segmento que compra más abiertamente artículos orientados hacia la salud y la belleza. De igual modo, un estudio realizado por Mintel mostró que los hombres más jóvenes son quienes están impulsando las ventas de productos y servicios de cuidado personal que tradicionalmente han sido vinculados con el público femenino, como manicure, tratamientos faciales y corporales (Marketingdirecto.com, 2013). Así mismo, actualmente el segmento masculino joven en países latinoamericanos, como el caso de México y Perú, tiene una mayor propensión a adquirir productos de carácter innovador, son más abiertos culturalmente y guían su actuar basados en principios más hedonistas y autónomos (Jiménez y San Martín, 2013; GfK, 2012). Esto concuerda con motivaciones de consumo orientadas con verse saludables y físicamente atractivos (Nizar y Nizar Souiden, 2009).

Frente a esta situación, se esperaría que la influencia negativa de la norma subjetiva en la intención de compra de productos de cuidado personal sea menor en el caso de los hombres jóvenes de países latinoamericanos con alto y moderado nivel de desarrollo de la categoría en cuestión. Esto se debería al mayor alejamiento de los valores tradicionales por parte de este segmento de la población masculina. A partir de ello, se desprenden las siguientes hipótesis.

- Hipótesis 3a: en países latinoamericanos con alto desarrollo de la categoría cuidado personal masculino, como en el caso de México, la influencia de la norma subjetiva en la intención de consumo de estos productos es menor entre los hombres más jóvenes.
- Hipótesis 3b: en países latinoamericanos con moderado desarrollo de la categoría cuidado personal masculino, como en el caso de Perú, la influencia de la norma subjetiva en la intención de consumo de estos productos es menor entre los hombres más jóvenes.

Pese a contar con una actitud positiva, la intención de compra puede estar limitada por la percepción de obstáculos existentes en el entorno que dificulten su concreción (Leeman y Ong, 2008; Chen, 2007). Al respecto, Yeon y Chung (2011) sugieren que la intención de comprar productos de cuidado de la piel y el cabello es mayor cuando los consumidores perciben más control sobre la compra. Esta percepción de control se refiere a la impresión de contar con los recursos y las capacidades necesarias para acceder a estos bienes.

En los últimos años, la oferta de productos y servicios de cuidado personal dirigida al público masculino se ha desarrollado considerablemente a partir de nuevos lanzamientos que amplían el abanico de soluciones existentes en el mercado latinoamericano (El Espectador, 2008). Esto con miras a satisfacer la demanda de los nuevos perfiles de consumidores varones preocupados por su imagen (Ruiz de Maya y Grande Esteban, 2006). Sin embargo, en cuanto al volumen, este tipo de productos dirigidos al segmento masculino aún cuenta con una reducida presencia en comparación con la oferta enfocada en el segmento femenino. De acuerdo con un estudio realizado por Mintel (2017), la mayoría de los productos de cuidado personal masculino cuentan con baja presencia en el sector retail. Al respecto, en América Latina, el volumen ofertado de productos de cuidado personal para varones representa $14.40 \%$ del volumen total de productos ofertados pertenecientes a la categoría belleza y cuidado personal (Euromonitor, 2017). 
Debido a la baja presencia que tienen los productos de la categoría en cuestión enfocados en el público masculino -comparado con aquellos que se enfocan en el público femenino - y el potencial de demanda de estos - basado en la consolidación de la clase media y en las nuevas preocupaciones de los hombres por su imagen-, se esperaría que los consumidores consideren aún limitada la oferta actual -en el contexto de volumenpara atender sus necesidades. Esta situación contribuiría a reducir la percepción de control para adquirir este tipo de productos.

- Hipótesis 4a: en países latinoamericanos con alto desarrollo de la categoría cuidado personal masculino, como en el caso de México, la percepción de control de los hombres influiría negativamente en la intención de consumo de estos productos.

- Hipótesis 4b: en países latinoamericanos con moderado desarrollo de la categoría cuidado personal masculino, como en el caso de Perú, la percepción de control de los hombres influiría negativamente en la intención de consumo de estos productos.

A continuación, se presenta el modelo conceptual y las hipótesis planteadas para esta investigación (Figura 2).

Figura 2. Hipótesis planteadas.

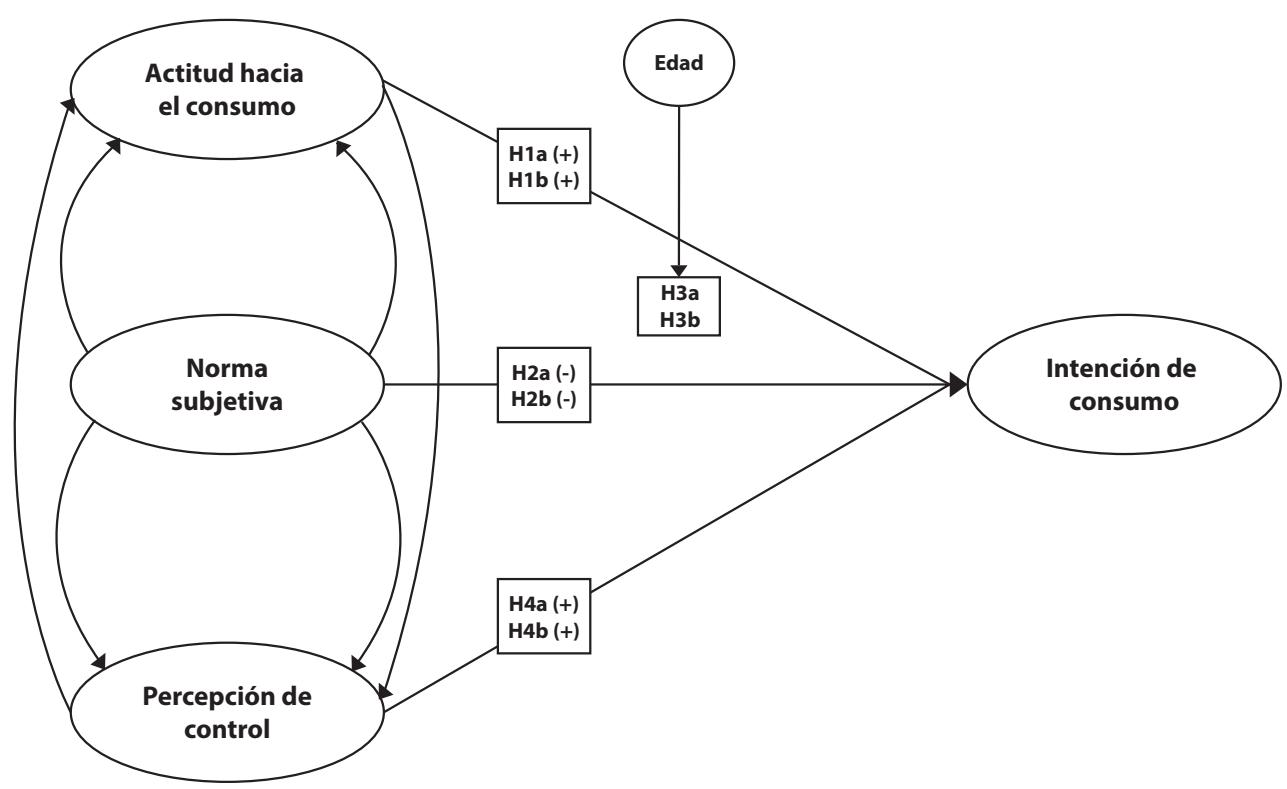

Fuente. Elaboración propia. 


\section{Metodología}

$\mathrm{C}^{1}$ instrumento de medición empleado fue Eun cuestionario, que se diseñó tomando en consideración tres factores independientes -actitud, norma subjetiva y percepción de contro- $\mathrm{y}$ un factor dependiente -intención por consumir en mayor medida los productos de cuidado personal-. Las escalas y afirmaciones fueron utilizadas en investigaciones realizadas en otros países y se adaptaron a los contextos locales donde se aplicó el instrumento (Januszewska y Viaene, 2001; Hoppe, Dutra de Barcellos, Marques Vieira y De Matos, 2012; Kim y Karpova, 2010).

Antes de salir a campo, el instrumento fue validado en el contexto de contenido a través de un panel de expertos bajo la técnica de consenso (Escobar-Pérez y Cuervo-Martínez, 2008). Para ello, se realizaron dos reuniones con cinco especialistas en investigación de mercados provenientes de ambos países. En estas reuniones, se discutió sobre la calidad de los ítems considerando su claridad para ser comprendidos y su coherencia, suficiencia y relevancia para explicar los constructos correspondientes. Previo a ello, se presentó el objetivo del estudio y se explicaron cada uno de los constructos involucrados, los ítems que los conforman y la forma en que iba a ser aplicado el cuestionario. Las recomendaciones surgidas en las reuniones fueron implementadas para mejorar el instrumento. Al final de la segunda reunión, los expertos llegaron a un consenso sobre la adecuada calidad de los ítems a partir de las consideraciones antes mencionadas.

La versión final del instrumento estuvo conformada por 11 afirmaciones que fueron evaluadas de manera individual a través del uso de escalas tipo Likert para medir el grado de aceptación o rechazo de los encuestados. Además, se incluyeron preguntas de control y de carácter demográfico. Los constructos analizados y las afirmaciones que las conforman se presentan más adelante (Tabla 1). 
Tabla 1. Constructos estudiados y afirmaciones que los componen.

\begin{tabular}{|c|c|c|}
\hline Constructos & & Afirmaciones \\
\hline \multirow{4}{*}{ Actitud } & act1 & Usar productos de cuidado personal resulta agradable para mí. \\
\hline & act2 & Usar productos de cuidado personal es algo varonil. \\
\hline & act3 & Usar productos de cuidado personal es algo que todo hombre debe hacer. \\
\hline & act4 & Usar productos de cuidado personal necesario para un hombre. \\
\hline \multirow{3}{*}{$\begin{array}{l}\text { Norma } \\
\text { subjetiva }\end{array}$} & ns1 & $\begin{array}{l}\text { Las personas cuya opinión valoro piensan que un hombre no debe } \\
\text { preocuparse mucho de los productos de cuidado personal que usan*. }\end{array}$ \\
\hline & ns2 & $\begin{array}{l}\text { Las personas cuya opinión valoro esperan que un hombre no use muchos } \\
\text { productos de cuidado personal*. }\end{array}$ \\
\hline & ns3 & $\begin{array}{l}\text { Mis amigos hombres y familiares cercanos hombres no se preocupan } \\
\text { mucho por los productos de cuidado personal que usan*. }\end{array}$ \\
\hline \multirow{2}{*}{$\begin{array}{l}\text { Percepción de } \\
\text { control }\end{array}$} & $\mathrm{pc} 1$ & $\begin{array}{l}\text { En mi país existen tantos productos de cuidado personal para hombres } \\
\text { como yo quisiera. }\end{array}$ \\
\hline & pc2 & $\begin{array}{l}\text { Es fácil encontrar productos de cuidado personal dirigidos a hombres como } \\
\text { yo. }\end{array}$ \\
\hline \multirow{2}{*}{$\begin{array}{l}\text { Intención de } \\
\text { consumo }\end{array}$} & ic1 & Estoy interesado en usar productos de cuidado personal. \\
\hline & ic2 & $\begin{array}{l}\text { Estoy interesado en usar más productos de cuidado personal de los que } \\
\text { uso ahora. }\end{array}$ \\
\hline
\end{tabular}

* Afirmaciones redactadas de forma negativa.

Fuente. Elaboración propia.

Cabe mencionar que durante el proceso de validez de contenido se definió que las afirmaciones que conforman el constructo norma subjetiva debían ser planteadas de forma negativa. Esto se debería a que la norma subjetiva se basa en la influencia del entorno y en las expectativas que el consumidor debe cubrir de acuerdo con su rol dentro de los grupos de pertenencia. En sociedades con fuerte presencia de valores tradicionales, como los analizados en este estudio, se espera que los hombres no consuman en exceso los productos pertenecientes a la categoría cuidado personal ni se preocupen en exceso por su imagen. Por ello, resulta más natural en este contexto comprender y procesar estas preguntas de forma negativa por parte de los encuestados.

El público objetivo de la investigación estuvo conformado por hombres de 18 a 60 años residentes en Guadalajara - Méxicoy Lima -Perú-. Tal como se mencionó en la introducción, se decidió estudiar estos dos países debido a que cuentan con realidades distintas en cuanto al desarrollo del mercado de belleza y cuidado personal dentro de la región latinoamericana. Al respecto, México cuenta con un mercado altamente desarrollado en el contexto de esta categoría. En 2016, el mercado de cuidado personal masculino en México alcanzó un valor de USD 1330 millones, superado solo por Brasil. Por su parte, el desarrollo de esta categoría en Perú aún es moderado en comparación con otros países de la región. El 
mercado peruano de productos de cuidado personal masculino alcanzó un valor USD 294 millones (Euromonitor, 2017). Además, en la última década, ambos países han consolidado su clase media, teniendo el poder adquisitivo suficiente para acceder a bienes y servicios con mayor valor agregado, como es el caso de los productos de belleza y cuidado personal (Salgado, Camarena y Díaz, 2015; Ipsos Perú, 2015). Las ciudades fueron seleccionadas por ser urbes que concentran una cantidad importante de habitantes y cuentan con un alto desarrollo económico (Istrate y Nadeau, 2012).
Los encuestados fueron individuos pertenecientes a los niveles socioeconómicos altos y medios con la finalidad de que en los resultados el factor poder adquisitivo no influya en la intención de consumo de esta categoría. En el caso de Guadalajara, se trabajó con varones de niveles $\mathrm{AB}-$ alta,$- \mathrm{C}+-$ media alta - y C - media - de acuerdo con la clasificación de la AMAI (2014). En el caso de Lima, se trabajó con los niveles A -alto-, B - medio alto - y C - medio bajo- de acuerdo con la Apeim (2014).

El tamaño de muestra en cada una de las ciudades fue de 300 casos distribuidos por

edad (Tabla 2).

Tabla 2. Distribución de la muestra por edad por tipo de mercado.

\begin{tabular}{|c|c|c|c|}
\hline Edades & $\begin{array}{c}\text { Mercado con moderado desarro- } \\
\text { llo de la categoría } \\
\text { (Guadalajara) (\%) }\end{array}$ & $\begin{array}{c}\text { Mercado con alto desarrollo de la } \\
\text { categoría } \\
\text { (Lima) (\%) }\end{array}$ & Total (\%) \\
\hline $18-24$ años & 25 & 23 & 24 \\
\hline $25-34$ & 28 & 30 & 29 \\
\hline $35-46$ & 26 & 27 & 20 \\
\hline $47-60$ & 20 & 20 & 27 \\
\hline
\end{tabular}

Fuente. Elaboración propia.

Los estudios que abordan una temática similar utilizando el modelo TPB o TRA han trabajado con muestras similares en el contexto de tamaño y representatividad (Yeon y Chung, 2011; Sukato y Elsey, 2009). Esto se debería al carácter académico de las investigaciones y a que se basan en una teoría ampliamente aplicada y aceptada. En estos casos, al igual que en este estudio, se busca priorizar la validez interna del modelo y de sus constructos, en sacrificio de la validez externa (Calder, Phillips y Tobout, 1982; Cook y Campbell, 1979).

El cuestionario fue aplicado de manera presencial por un equipo de encuestadores. El trabajo de campo tuvo una duración de cinco semanas, durante los meses de junio y julio de 2015. Para garantizar la compresión unívoca por parte de los encuestados acerca de la categoría evaluada - productos de cuidado personal dirigidos al sector masculino-, se les presentó previamente una definición estándar que incluía ejemplos con imágenes de productos que la conforman. Dichos productos fueron recopilados de diferentes países de la región. Así, se incluyeron tanto productos que tradicionalmente usa el consumidor de ambos países -champú unisex, gel, reacondicionador, perfume, crema para afeitar, talco, aftershave, colonia de baño, 
peluquería unisex y sauna-, como productos más innovadores - champú especializado por tipo de cabello, cremas para la piel, cremas para peinar, tratamientos faciales, tratamiento para el cabello y depilación-. Tras recibir confirmación de los encuestados respecto de comprender la amplitud de la categoría y cómo se relacionan estos productos con su propia experiencia de consumo, se inició la aplicación del instrumento.

\section{Resultados}

$\mathrm{C}$ on el fin de preparar los datos para el posterior análisis, se recodificaron de forma inversa los valores obtenidos en las afirmaciones que conforman el constructo norma subjetiva, dado que fueron formulados de forma negativa para el cuestionario. Esta práctica ha sido aplicada en otros estudios (Brakus, Schmitt y Zarantonello, 2009). La recodificación inversa de estos valores permitió evaluar, bajo la misma direccionalidad, la influencia de la actitud, norma subjetiva y percepción de control sobre la intención de consumo y mejorar la confiabilidad (Lee, Murphy y Swilley, 2009). Contando con la misma direccionalidad, un coeficiente positivo en cualquiera de las tres variables dependientes implicaría una influencia positiva sobre la intención de consumo, mientras que un coeficiente negativo implicaría una influencia negativa sobre este.

Dado que la investigación busca examinar las relaciones existentes entre constructos de carácter multivariado como la actitud hacia el consumo, norma subjetiva, percepción de control e intención de consumo, se llevó a cabo un análisis factorial exploratorio para corroborar la existencia de estos factores y posteriormente se utilizaron ecuaciones estructurales para probar el modelo propuesto teóricamente. La pertinencia de utilizar un modelo de ecuaciones estructurales en este estudio se debe a que dicha herramienta permite analizar el impacto de múltiples relaciones simultáneas entre constructos complejos representados por diferentes variables de medición (Harris y Goode, 2004; Hair, Black, Babin y Anderson, 2010).

Los índices de bondad del modelo propuesto exceden los niveles de aceptación sugeridos por estudios previos ( $\mathrm{X}^{2}$ (38) $=81.29$, CFI $=0.99$, TLI $=0.98$, RMSEA $=0.04)$, lo cual refleja su adecuado nivel de ajuste (Jöreskog y Sorbom, 1992). Tal como se observa (Tabla 3), la fiabilidad de cada constructo está confirmada al obtener valores superiores a 0.70 en el alpha de Cronbach (Nunnally y Bernstein, 1994). La validez convergente se confirma mediante el examen de la varianza media extraída, las elevadas cargas factoriales obtenidas en cada constructo y los índices de bondad del ajuste. Los valores de la varianza media extraída de todos los constructos se encuentran por encima del nivel recomendado (0.50) $\mathrm{y}$ todos los constructos en el modelo de medición tienen cargas factoriales que exceden a 0.6 y son significativas $(\mathrm{p}<0.05)$, $\mathrm{y}$ así mostrar ser apropiados para el estudio (Bagozzi y Yi, 1988). Los índices de ajuste del modelo Comparative Fit Index (CF), TuckerLewis index (TLI) exceden el 0.90, teniendo valores aceptables (Anderson y Gerbing, 1988). Además, los autores encontraron que todas las covarianzas al cuadrado entre los 
factores eran más bajas que el valor de la varianza media extraída, de acuerdo con lo sugerido por Fornell y Larcker (1981), y así aceptar los valores discriminantes de los constructos. Por tanto, los constructos del modelo de medición presentado en este estudio poseen una adecuada fiabilidad y validez.

Tabla 3. Cargas factoriales, análisis de fiabilidad y valores discriminantes.

\begin{tabular}{|c|c|c|c|c|c|c|c|}
\hline $\begin{array}{c}\text { Variable latente } \\
\text { (factor) }\end{array}$ & Ítems & $\begin{array}{c}\text { Carga } \\
\text { factorial }\end{array}$ & p-valor & $\begin{array}{l}\text { Alpha de } \\
\text { Cronbach }\end{array}$ & Ave & $\mathbf{R}^{2}$ & $\begin{array}{c}\text { Valores } \\
\text { discriminantes* }\end{array}$ \\
\hline \multirow{4}{*}{ Actitud } & act 1 & 0.76 & 0.000 & \multirow{4}{*}{0.856} & \multirow{4}{*}{0.605} & \multirow{4}{*}{$\begin{array}{l}\text { ACT-NS: } 0.221 \\
\text { ACT-PC: } 0.073 \\
\text { ACT-IC: } 0.360\end{array}$} & \multirow{4}{*}{ Cumple } \\
\hline & act2 & 0.83 & 0.000 & & & & \\
\hline & act3 & 0.74 & 0.000 & & & & \\
\hline & act 4 & 0.78 & 0.000 & & & & \\
\hline \multirow{3}{*}{ Norma subjetiva** } & ns1 & 0.88 & 0.000 & \multirow{3}{*}{0.881} & \multirow{3}{*}{0.724} & \multirow{3}{*}{$\begin{array}{l}\text { NS-ACT: } 0.221 \\
\text { NS-PC: } 0.168 \\
\text { NS-IC: } 0.336\end{array}$} & \multirow{3}{*}{ Cumple } \\
\hline & ns2 & 0.91 & 0.000 & & & & \\
\hline & ns3 & 0.76 & 0.000 & & & & \\
\hline \multirow{2}{*}{$\begin{array}{l}\text { Percepción de } \\
\text { control }\end{array}$} & pc1 & 0.88 & 0.000 & \multirow{2}{*}{0.811} & \multirow{2}{*}{0.687} & \multirow{2}{*}{$\begin{array}{l}\text { PC-ACT: } 0.073 \\
\text { PC-NS: } 0.168 \\
\text { PC-IC: } 0.137\end{array}$} & \multirow{2}{*}{ Cumple } \\
\hline & $\mathrm{pc} 2$ & 0.78 & 0.000 & & & & \\
\hline \multirow{2}{*}{$\begin{array}{l}\text { Intención de } \\
\text { consumo }\end{array}$} & ic1 & 0.97 & 0.000 & \multirow{2}{*}{0.841} & \multirow{2}{*}{0.757} & IC-ACT: 0.360 & \multirow{2}{*}{ Cumple } \\
\hline & ic2 & 0.76 & 0.000 & & & IC-PC: 0.137 & \\
\hline
\end{tabular}

* Cumplimiento de la prueba de valores discriminantes: Ave > R2

** Constructo norma subjetiva que considera los valores recodificados de forma inversa.

Fuente. Elaboración propia.

Las hipótesis son puestas a prueba mediante el modelo de ecuaciones estructurales que fue analizado haciendo uso del paquete estadístico Stata y la moderación a través de Process

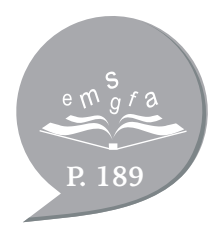
(Hayes, 2013). Los resultados del modelo en cuanto a relaciones causales, coeficientes estructurales estandarizados y niveles de confianza se presentan más adelante (Tabla 4). 
Tabla 4. Resultados del modelo propuesto.

\begin{tabular}{|l|l|l|l|l|l|l|l|}
\hline Hipótesis & \multicolumn{1}{|c|}{ País } & \multicolumn{1}{|c|}{ Relación causal } & Coeficiente & \multicolumn{1}{c|}{$\begin{array}{c}\text { Error } \\
\text { estándar }\end{array}$} & p-value & \multicolumn{2}{c|}{$\begin{array}{c}\text { Intervalo de } \\
\text { confianza 95 \% }\end{array}$} \\
\hline H1a & M. alto desarrollo & AC --> IC & 0.548 & 0.053 & 0.000 & 0.444 & 0.654 \\
\hline H1b & $\begin{array}{l}\text { M. moderado } \\
\text { desarrollo }\end{array}$ & AC --> IC & 0.364 & 0.058 & 0.000 & 0.249 & 0.479 \\
\hline H2a & M. alto desarrollo & NSr* --> IC & -0.259 & 0.058 & 0.000 & -0.376 & -0.145 \\
\hline H2b & $\begin{array}{l}\text { M. moderado } \\
\text { desarrollo }\end{array}$ & NSr* --> IC & -0.532 & 0.059 & 0.000 & -0.648 & -0.416 \\
\hline H3a & M. alto desarrollo & $\begin{array}{l}\text { Intercepto } \\
\text { NSr* x Edad --> IC }\end{array}$ & -0.009 & 0.004 & 0.008 & -0.017 & -0.003 \\
\hline H3b & $\begin{array}{l}\text { M. moderado } \\
\text { desarrollo }\end{array}$ & $\begin{array}{l}\text { Intercepto } \\
\text { NSr* } \text { Edad --> IC }\end{array}$ & -0.009 & 0.003 & 0.002 & -0.016 & -0.004 \\
\hline H4a & M. alto desarrollo & PC --> IC & -0.090 & 0.063 & 0.015 & -0.213 & 0.035 \\
\hline H4b & $\begin{array}{l}\text { M. moderado } \\
\text { desarrollo }\end{array}$ & PC --> IC & -0.176 & 0.067 & 0.009 & -0.308 & -0.044 \\
\hline
\end{tabular}

* Constructo norma subjetiva que considera los valores recodificados de forma inversa.

Fuente. Elaboración propia.

Los resultados obtenidos reflejan que la actitud -ACT - hacia el consumo de productos de belleza y cuidado personal es el factor que cuenta con la mayor influencia positiva hacia la intención de consumo. Esto ocurre tanto en la muestra de consumidores provenientes del mercado con alto nivel de desarrollo de la categoría $(\lambda=0.548)$ como en la muestra proveniente del mercado con moderado nivel de desarrollo $(\lambda=0.364)$, contando con valores significativos en ambos casos $(\mathrm{p}<0.05)$. Estos resultados respaldan las hipótesis 1a y $1 \mathrm{~b}$.

La norma subjetiva que considera los valores recodificados de forma inversa de las afirmaciones que la conforman $-\mathrm{NSr}-$ presenta una influencia negativa hacia la intención de consumo de los productos en cuestión. Esto ocurre tanto en la muestra de consumidores provenientes del mercado con alto nivel de desarrollo de la categoría $(\lambda=-0.259)$ como en la muestra proveniente del mercado con moderado nivel de desarrollo $(\lambda=-0.532)$, contando ambos casos con resultados significativos $(p<0.05)^{4}$. Estos resultados respaldan las hipótesis $2 \mathrm{a}$ y $2 \mathrm{~b}$.

Los valores obtenidos por el intercepto NSr $\mathrm{x}$ edad indican que el impacto total generado por la edad reduciría la influencia negativa de la norma subjetiva. Esto ocurre tanto en la muestra de consumidores provenientes del mercado con alto nivel de desarrollo de

\footnotetext{
${ }^{4}$ Cabe recordar que las afirmaciones que conforman la norma subjetiva fueron redactadas de forma negativa en el cuestionario con miras a lograr una comprensión y procesamiento más natural de ellos por parte de los encuestados. Posteriormente, los valores obtenidos fueron recodificados de forma inversa y así conseguir la misma direccionalidad que los constructos actitud y percepción de control. De esta manera, los coeficientes negativos obtenidos en el factor norma subjetiva implicarían una influencia negativa en la intención de consumo.
} 
la categoría $(\lambda=-0.009)$ como en la muestra proveniente del mercado con moderado nivel de desarrollo $(\lambda=-0.009)$, contando en ambos casos con resultados significativos $(\mathrm{p}<0.05)$. La siguiente información (Tabla 3; Tabla 4), desarrollada en Process, muestra de manera gráfica la influencia de la edad en la relación norma subjetiva-intención de consumo para tres niveles de edades. En ellos se observa, que conforme la edad es menor, la pendiente de la relación en cuestión se reduce. Estos resultados respaldan las hipótesis 3a y 3b.
La percepción de control (PC) presenta una influencia negativa hacia la intención de consumo de los productos en cuestión. Esto ocurre en la muestra proveniente del mercado con moderado nivel de desarrollo de la categoría $(\lambda=-0.176)$. Este resultado tiene un valor significativo $(\mathrm{p}<0.05)$ que respalda la hipótesis $4 \mathrm{~b}$. El resultado obtenido de la muestra proveniente del mercado con alto nivel de desarrollo de la categoría presenta una tendencia similar $(\lambda=-0.090)$, sin embargo, el valor no es significativo $(p>0.05)$ por lo cual no permite respaldar la hipótesis $4 \mathrm{a}$.

Figura 3. Influencia de la edad en la relación noma subjetiva-intención de consumo (mercado con alto desarrollo de la categoría cuidado personal masculino).

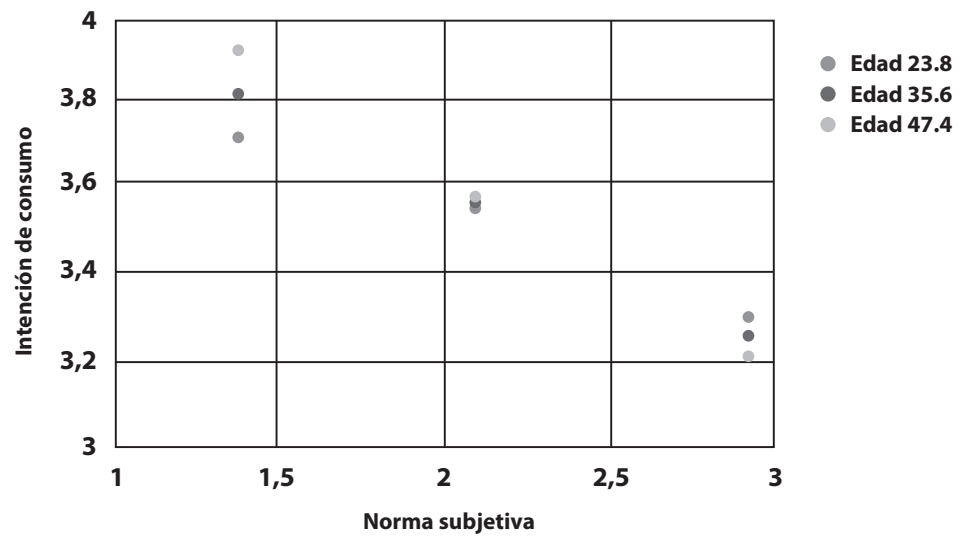

Fuente. Elaboración propia.

Figura 4. Influencia de la edad en la relación noma subjetiva-intención de consumo - mercado con moderado desarrollo de la categoría cuidado personal masculino-.

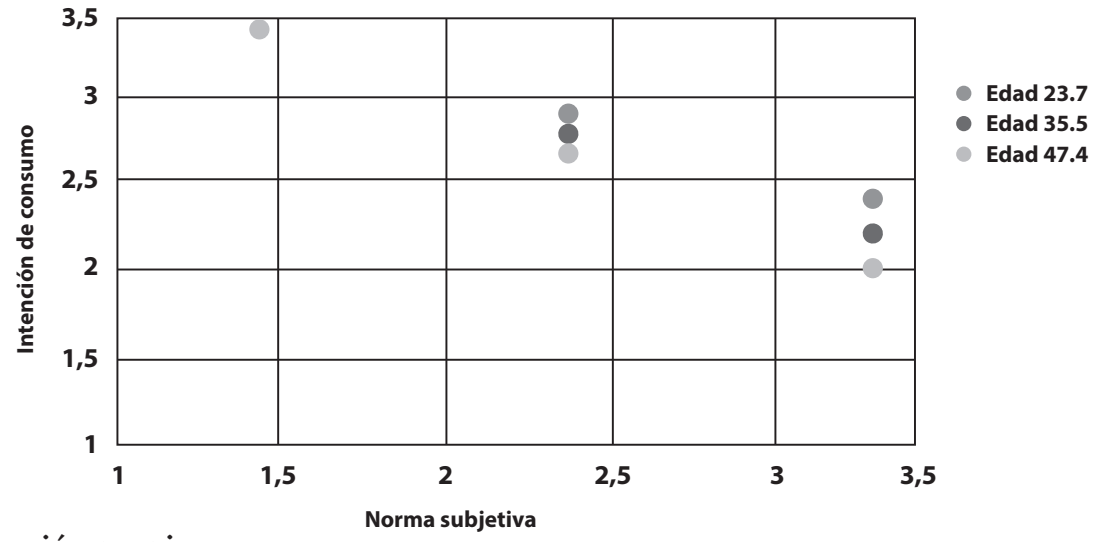

Fuente. Elaboración propia. 


\section{Conclusiones}

Tos resultados de la investigación muestran Lque los factores analizados impactan de distinta manera en la intención de consumo de los productos de cuidado personal masculino en América Latina, y favorecen o inhiben finalmente el consumo real de estos. Al respecto, la actitud hacia los productos de cuidado personal masculino es el factor que tiene el mayor impacto positivo en la intención de consumo, lo cual daría indicios del potencial de crecimiento de la demanda por estos productos. Tales resultados guardan relación con el proceso de desarrollo de valores de autoexpresión en la población latinoamericana (World Values Survey, 2017; Inglehart, Basanez, Diez-Medrano, Halman y Luijkx, 2004). Estos valores refuerzan la necesidad de construir una identidad propia que se refleja en una mayor preocupación por la imagen personal y un incremento en la intención de consumo de productos asociados con su cuidado (Sarpila, 2012; Featherstone, 2007; Ruiz de Maya y Grande Esteban, 2006). De igual manera, estos valores favorecen la tolerancia y la igualdad hacia las expresiones de género, como el consumo por parte de los hombres de ciertos productos definidos culturalmente para un público exclusivamente femenino (Inglehart et al., 2004).

La norma subjetiva sería el principal factor que inhibe el consumo de los productos en cuestión dentro del segmento masculino. Al respecto, el consumidor varón de América Latina consideraría que las personas que conforman su entorno rechazarían el hecho de que ellos usen estos productos. El impacto negativo de la norma subjetiva se explicaría a partir de la presencia de valores tradicionales dentro de la región y una mayor presión del entorno sobre el individuo (World Values Survey, 2017). Las sociedades donde priman estos factores ponen más énfasis en las tradiciones y el respeto a las creencias establecidas (Inglehart et al., 2004). Al respecto, el uso de este tipo de productos iría en contra del comportamiento masculino esperado. Un reflejo de estos valores tradicionales son las creencias machistas, según las cuales los hombres no deberían usar ciertos tipos de productos, como cosméticos, dado que puede verse amenazada su masculinidad (Souiden y Diagne, 2009).

Por otra parte, la norma subjetiva influiría en menor medida en el caso de los consumidores jóvenes. Estos resultados reflejarían la presencia de valores más cercanos al hedonismo, autonomía, apertura cultural y orientación al éxito dentro de estos segmentos de la población (Getrich et al., 2012; GfK, 2012; Jiménez y San Martín, 2013). Estos valores generarían una mayor predisposición a aceptar este tipo de productos, debido a que concuerdan con motivaciones, como verse saludables, físicamente atractivos, jóvenes y preocupados por su estética (Nizar y Nizar Souiden, 2009).

El impacto de la percepción de control hacia la intención de consumo es negativo, pero de menor influencia que el de norma subjetiva para el mercado con moderado nivel de desarrollo de la categoría cuidado personal masculino. Esto se encontraría alineado con la baja presencia de estos productos en comparación con aquellos enfocados en el público femenino. Se esperaría que, conforme la categoría continúe desarrollándose, el impacto de la percepción de control se torne positivo. Los resultados vinculados con la percepción de control no son significativos para el mercado con alto nivel de desarrollo de la categoría en cuestión. 
Los resultados obtenidos en los factores actitud y norma subjetiva se alinean a estudios como los de Ntibagirirwa (2009) y Uy (2009), donde se sostiene que los valores y las creencias de una comunidad pueden influir en resultados económicos, como vendría a ser en este caso el desarrollo de una categoría de productos.
A partir de estos hallazgos, se propone redefinir las propuestas de valor de las marcas de productos de cuidado personal orientadas al segmento masculino en América Latina. Estas marcas deben reemplazar los beneficios enfocados en belleza como eje de la comunicación dirigida hacia el público masculino, por otros más acordes con valores propios de sociedades tradicionales, tales como el éxito.

\section{Limitaciones y futuras investigaciones}

Eque podrán ser aprovechadas como oportunidades para el desarrollo de futuras investigaciones.

Respecto del modelo propuesto, este centra su atención en la intención de consumo de productos de cuidado personal masculino, mas no en el consumo en sí mismo. Si bien la literatura demuestra que la intención de consumo es un buen predictor del consumo per se (Ajzen, 1991), las futuras investigaciones podrían incluir a esta última variable en el modelo para analizar los factores que influyen directamente en ella sin mediación de la intención. De igual manera, las futuras investigaciones podrían complementar el conocimiento sobre el consumo de esta categoría e incluir dentro del modelo propuesto nuevos factores como los valores predominantes en diferentes segmentos de consumidores o la asociación de la marca con el público femenino.
Respecto de la categoría cuidado personal masculino, este estudio la considera como un todo, abarcando dentro de ella algunos productos que han sido vinculados tradicionalmente al segmento masculino - crema para afeitar, talco, aftershave, colonia de baño-, y otros al segmento femenino - tratamientos faciales, tratamiento para el cabello, depilación-. Las futuras investigaciones podrían complementar los resultados de este estudio analizando tal diferencia.

Con relación al diseño metodológico, si bien esta investigación analiza dos contextos distintos en cuanto al desarrollo de la categoría cuidado personal masculino en América Latina - uno con alto y otro con moderado nivel de ventas - , esto podría ser considerado como una limitación al tratar de abarcar las particularidades existentes dentro de la región. Al respecto, las futuras investigaciones podrían ampliar el ámbito de estudio a otros países y ciudades latinoamericanas con miras a buscar una mayor representatividad, 
considerando otras características adicionales a las ventas para su elección, como el nivel de machismo, la adopción de nuevas tendencias de consumo o la preocupación por la imagen personal existente en cada país.

Por otra parte, la importancia de la norma subjetiva identificada en este estudio da indicios para sospechar que las respuestas obtenidas a través de encuestas que aborden esta temática pueden ser influenciadas en cierta medida por las expectativas sociales. Según ello, las futuras investigaciones deberán analizar el condicionamiento de la deseabilidad social en las respuestas a través del uso de la escala de Marlowe-Crowne, la cual ha sido ampliamente validada para dicho fin (Gutiérrez, Espinosa, Gesteira y García-Vera, 2016).

Finalmente, se abre la posibilidad de complementar los resultados obtenidos en este estudio con futuras investigaciones basadas en diseños experimentales. Esto permitiría analizar el comportamiento de consumo real de la categoría en cuestión dentro de situaciones simuladas, controlando a priori factores externos, como el sesgo generado por la deseabilidad social.

\section{Referencias}

Ajzen, I y Fishbein, M. (1980). Understanding attitudes and predicting social behavior. New Jersey: Prentice Hall.

Ajzen, I. (1991). The theory of planned behavior. Organizational Behavior and Human Decision Processes, 50, 179-211.

Ajzen, I. (2002). Residual effects of past on later behavior: Habituation and reasoned action perspectives. Personality and Social Psychology Review, 6(2), 107-122.

Ajzen, I. (2011). The theory of planned behaviour: Reactions and reflections. Psychology y Health, 26(9), 1113-1127.

Ajzen, I. (2014). The theory of planned behavior is alive and well, and not ready to retire: A commentary on Sniehotta, Presseau, and AraújoSoares. Health Psychology Review, 9(2), 131-137.

AMAI (Asociación Mexicana de Inteligencia de Mercado y Opinión) (2014). Niveles socioeconómicos. Recuperado de http://nse.amai.org/data/

Anderson, J. y Gerbing, D. (1988). Structural equation modeling in practice: A review and recommended two-step approach. Psychological Bulletin, 103 (3), 411-423.
Ang, M., Ramayah, T. y Amin, H. (2015). Efficacy of the theory of planned behavior in the context of hiring Malaysians with disabilities. Journal of Developing Areas, 49(3), 3-25.

Apeim (Asociación Peruana de Empresas de Investigación de Mercados) (2014). Niveles socioeconómicos 2014. Recuperado de http://www. apeim.com.pe/wp-content/themes/apeim/docs/ nse/Apeim-NSE-2014.pdf

Bagozzi, R. y Yi, Y. (1988). On the evaluation of structural equation models. Journal of the Academy of Marketing Science, 16, 74-94.

Berg, L. y Teigen, M. (2008). Gendered consumer competences in households with one vs two adults. International Journal of Consumer Studies, $33(1), 31-41$.

Brakus, J. J., Schmitt, B. H. y Zarantonello, L. (2009). Brand experience: What is it? How is it measured? Does it affect loyalty? Journal of Marketing, 73(3), 52-68.

Buttle, F. y Bok, B. (1996). Hotel marketing strategy and the theory of reasoned Action. International Journal of Contemporary Hospitality Management, 8(3), 5-10. 
Calder, B., Phillips, L. y Tybout, A. (1982). The concept of external validity. Journal of Consumer Research, 9, 240-244.

Carpi, A., Breva, S. y Palermo, F. (2005). La teoría de la acción planeada y la reducción del estrés percibido para prevenir la enfermedad cardiovascular. Anales de Psicología, 21(1), 84-91.

Chen, M. (2007). Consumer attitudes and purchase intentions in relation to organic foods in Taiwan: Moderating effects of food-related personality traits. Food Quality and Preference, 18(7), 1008-1021.

Chen, S. y Li, S. (2010). Consumer adoption of e-service: Integrating technology readiness with the theory of planned behavior. African Journal of Business Management, 4(16), 3556-3563.

Chiou, J. (1998). The effects of attitude, subjective norm, and perceived behavioral control on consumers' purchase intentions: The moderating effects of product knowledge and attention to social comparison information. Proceedings of the National Science Council, Republic of China, 9(2), 298308.

Coley, A. y Burgess, B. (2003), Gender differences in cognitive and affective impulse buying. Journal of Fashion Marketing and Management, 7(3), 282-95.

Cook, T. y Campbell, D. (1979). Quasi-experimentation: Design and analysis issues for field setting. Chicago: Rand McNally.

Dano, F., Roux, E. y Nyeck, S. (2003), Les hommes, leur apparence et les cométiques: Approche sociosémiotique. Décisions Marketing, 29, 7-18.

Diekman, A. B., Eagly, A. H., Mladinic, A. y Ferreira, M. C. (2005). Dynamic stereotypes about women and men in Latin America and the United States. Journal of Cross-Cultural Psychology, 36(2), 209-226.

El Espectador (14 junio 2008). Vanidad masculina hecha industria. Recuperado de https://www.elespectador. com/impreso/negocios/articuloimpreso-vanidadmasculina-hecha-industria

Escobar-Pérez, J. y Cuervo-Martínez, A. (2008). Validez de contenido y juicio de expertos: una aproximación a su utilización. Avances en Medición, 6, 27-36.

Euromonitor (2016). Consumer lifestyles in Mexico.
Euromonitor (2017). Consumo per capita de productos de cuidado personal masculino a precios constantes.

Farah, M. (2017). Consumers' switching motivations and intention in the case of bank mergers: A cross-cultural study. International Journal of Bank marketing, 35(2), 254-274.

Featherstone, M. (2007). Consumer culture and postmodernism (2. ${ }^{\mathrm{a}}$ ed.). Londres: Sage.

Fila, S. A. y Smith, C. (2006). Applying the theory of planned behavior to healthy eating behaviors in urban Native American youth. International Journal of Behavioral Nutrition and Physical Activity, $3(1)$.

Fishbein, M. \& Ajzen, I. (1981). Attitudes and voting behavior: An application of the theory of reasoned action. In G. M. Stephenson \& J. M. Davis (Eds.), Progress in Applied Social Psychology (Vol. I, pp. 253 -313). London: Wiley.

Fornell, C. y Larcker, D. (1981). Evaluating structural equation models with unobservable variables and measurement error. Journal of Marketing Research, $18,39-50$.

Getrich, C., Sussman, A., Helitzer, D., Hoffman, R., Warner, T., Sánchez, V., Solares, A. y Rhyne, R. (2012). Expressions of machismo in colorectal cancer screening among New Mexico Hispanic subpopulations. Qualitative Health Research, 22 (4), 546-559.

GfK: "Mentalidad triunfadora de consumidores en el Perú es similar a la de China" (26 octubre 2012). Recuperado de https://gestion.pe/noticias-deroper-valuescope-11299

Gill, R., Henwood, K. y McLean, C. (2005). Body projects and regulation of normative masculinity, Body y Society, 11(1), 37-56.

Gutiérrez, J., Espinosa, R., Gesteira, C. y GarcíaVera, M. (2016). La escala de deseabilidad social de Marlowe-Crowne: baremos para la población general española y desarrollo de una versión breve. Anales de Psicología, 32(1), 206-217.

Gutmann, M. C. (ed.) (2003). Changing men and masculinities in Latin America (2. ${ }^{\mathrm{a}}$ ed.). Londres: Duke University Press. 
Hair, J., Black, W., Babin, B. y Anderson, R. (2010). Multivariate data analysis: A global perspective ( $7 .^{\mathrm{a}}$ ed.). Upper Saddle River: Pearson.

Harris, L. C. y Goode, M. M. (2004). The four levels of loyalty and the pivotal role of trust: a study of online service dynamics. Journal of Retailing, 80(2), $139-158$

Hayes, A. (2013). Introduction to mediation, moderation, and conditional process analysis. Nueva York: The Guilford Press.

Hillhouse, J. J., Turrisi R. y Kastner, M. (2000). Modeling tanning salon behavioral tendencies using appearance motivation, self-monitoring and the theory of planned behavior. Health Education Research, 15(4), 405-14.

Hoppe, A., Dutra de Barcellos, M., Marques Vieira, L. y Matos, C. A. de (2012). Comportamento do consumidor de produtos orgânicos: uma aplicação da teoria do comportamento planejado. Revista de Administração e Contabilidade da Unisinos, 9(2), 174188.

Inglehart, R. y Baker, W. (2000). Modernization, cultural change, and the persistence of traditional values. American Sociological Review, 65, 19-51.

Inglehart, R., Basanez, M., Diez-Medrano, J., Halman, L. y Luijkx, R. (2004). Human Beliefs and Values: A cross-cultural sourcebook based on the 1999-2002 values surveys. México: Siglo XXI.

Ipsos Perú (2013). Perfil del adulto joven. Recuperado de https://www.ipsos.com/es-pe/perfil-del-adultojoven-2013

Ipsos Perú (2015). Liderazgo en productos comestibles 2015. Recuperado de https://www.ipsos.com/espe/liderazgo-en-productos-comestibles-2015

Istrate, E. y Nadeau, C. A. (30 noviembre 2012). Global MetroMonitor 2012:

Slowdown, Recovery, and Interdependence. En Brookings. Recuperado de https://www.brookings. edu/research/global-metromonitor-2012slowdown-recovery-and-interdependence/

Jain, S., Khan, M. y Mishra, S. (2017). Understanding consumer behavior regarding luxury fashion goods in India based on the theory of planned behavior. Journal of Asia Business Studies, 11(1), 4-21.
Jalilvand, M. y Samiei, N. (2012). The impact of electronic word of mouth on a tourism destination choice: Testing the theory of planned behavior (TPB). Internet Research, 22(5), 591-612.

Januszewska, R. y Viaene, J. (2001). Application of the theory of planned behavior to consumption of chocolate: Cultural differences across Belgium and Poland. Journal of Euromarketing, 10(2), 1-26.

Jiménez, N. H. Torres y San Martín Gutiérrez, S. (2013). El perfil psico-sociológico y demográfico del consumidor mexicano. Estudios Demográficos y Urbanos, 28(3), 681-710.

Jöreskog, K.G. y Sörbom, D. (1992). LISRELL VIII. Analysis of linear structural relations. Mooresville, IN: Scientific Software.

Kautonen, T., Gelderen, M. van y Fink, M. (2015). Robustness of the theory of planned behavior in predicting entrepreneurial intentions and actions. Entrepreneurship Theory and Practice, 39(3), 655674.

Khan, I., Dongping, H., Abdullah, M., Ahmad, Z., Ahmad Ghauri, T. y Ghazanfar, S. (2017). Men's attitude and motivation toward consumption of grooming products: A comparison of Chinese and Pakistani male consumers. Cogent Business $y$ Management, 4(1), 1-14.

Kim, H. y Karpova, E. (2010). Consumer attitudes toward fashion counterfeits: Application of the theory of planned behavior. Clothing and Textiles Research Journal, 28(2), 79-94.

Lee, C. y Green, R. T. (1991). Cross-cultural examination of the Fishbein behavioral intentions model. Journal of International Business Studies, 22(2), 289-305.

Lee, H. Y., Qu, H. y Kim, Y. S. (2007). A study of the impact of personal innovativeness on online travel shopping behavior: A case study of Korean travelers. Tourism Management, 28(3), 886-897.

Lee, R., Murphy, J. y Swilley, E. (2009). The moderating influence of hedonic consumption in an extended theory of planned behaviour. The Service Industries Journal, 29 (4), 539-555.

Leeman, O. y Ong, J. (2008). Lost and found again: Subjective norm in gym membership. DLSU Business y Economics Review, 18(1), 13-27. 
Marketingdirecto.com (2013). A los hombres no les interesan los productos de belleza en las tiendas, pero son grandes compradores online. Recuperado de https:// www.marketingdirecto.com/digital-general/ digital/a-los-hombres-no-les-interesan-losproductos-de-belleza-en-las-tiendas-pero-songrandes-compradores-online

Mintel (10 septiembre 2013). Beauty and personal care product launches targeted to men increase by $70 \%$ over the past six years. Recuperado de http://www.mintel.com/press-centre/beautyand-personal-care/beauty-personal-care-productlaunches-increase-substantially

Mintel (30 enero 2017). Male beauty gains momentum. Recuperado de http://www.mintel.com/blog/ beauty-market-news/male-beauty-gainsmomentum

Navarro, A. y Delgado, E. (2006). Los nuevos valores masculinos, del hombre tradicional al "metrosexual". En: S. Ruiz de Maya and G. Idelfonso, ed., Comportamientos de compra del consumidor 29 casos reales, 1ra ed. Madrid: ESIC Editorial, pp.131-138.

Netemeyer, R. G., Burton, S. y Johnston, M. (1991). A comparison of two models for the prediction of volitional and goal-directed behaviors: A confirmatory analysis approach. Social Psychology Quarterly, 54(2), 87-100.

Nizar, S. y Nizar Souiden, M. D. (2009). Canadian and French men's consumption of cosmetics: A comparison of their attitudes and motivations. Journal of Consumer marketing, 26(2), 97-109.

Ntibagirirwa, S. (2009). Cultural values, economic growth and development. Journal of Business Ethics, $84,297-311$.

Nunnally, J. C. y Bernstein, I. (1994). Psychometric theory. Nueva York: McGraw-Hill.

Räsänen, P. y Wilska, T. (2007). Finnish students' attitudes towards commercialised sex. Journal of Youth Studies, 10(5), 557-75.

Reardon, C. A. y Govender, K. (2013). Masculinities, cultural worldviews and risk perceptions among South African adolescent learners. Journal of Risk Research, 16(6), 753-770.
Salgado Beltrán, L., Camarena Gomez, D. M. y Diaz Leon, J. (2016). The Mexican consumer, reluctant or receptive to new foods? British Food Journal, 118(3), 734-748.

Sarpila, O. (2012). I am not spending on my appearance! Examining self-evaluated low-level consumers of clothing and beauty care in Finland, 1999-2009. International Review of Social Research, 2(2), 53-72.

Sarpila, O. y Räsänen, P. (2011). Personal care consumption in finland: Trends in the early 2000s. The International Journal of Sociology and Social Policy, 31(7), 441-455.

Schwartz, S. H. (1992). Universals in the content and structure of values: Theory and empirical tests in 20 countries. En M. Zanna (ed.), Advances in experimental social psychology (vol. 25, pp. 1-65). Nueva York: Academic Press.

Sheth, J. N. (1974). A theory of family buying decisions. Recuperado de http://www.jagsheth.com/wpcontent/uploads/2014/02/a_theory_of_family_ buying_decisions.pdf

Shih, Y.-Y. y Fang, K. (2004). The use of a decomposed theory of planned behavior to study Internet banking in Taiwan. Internet Research, 14(3), 13-223.

Shilling, C. (2003). The body and social theory (2. ${ }^{\mathrm{a}}$ ed.). Londres: Sage.

Souiden, N. y Diagne, M. (2009). Canadian and French men's consumption of cosmetics: A comparison of their attitudes and motivations. Journal of Consumer marketing, 26(2), 97-109.

Sukato, N. y Elsey, B. (2009). A model of male consumer behaviour in buying skin care products in Thailand. ABAC Journal, 29 (1), 39-52.

Tournois, L. (2014). Too many products? Reaching the next billion customers of the beauty market. Journal of Business Strategy, 35(5), 3-13.

Turner, B. (2008). The body and society (3. ${ }^{\mathrm{a}}$ ed.). Londres: Sage.

Uy, A. O. (2009). Can culture explain economic growth? A note on issues regarding culture-growth studies. Journal of Economics and Economic Education Research, 10(3), 85-105. 
Wing Kwan, M. Y., Bray, S. R. y Martin Ginis, K. A. (2009). Predicting physical activity of first-year university students: An application of the theory of planned behavior. Journal of American College Health, 58(1), 45-55.

World Values Survey (2017). WVS Wave 6 2010-2014. Recuperado de http://www.worldvaluessurvey. org/WVSDocumentationWV6.jsp

Yadav, R. y Pathak, G. (2017). Determinants of consumers' green purchase behavior in a developing nation: Applying and extending the theory of planned behavior. Ecological Economics, 134, 114-122.

Yeon Kim, H. y Chung, J. E. (2011). Consumer purchase intention for organic personal care products. Journal of Consumer Marketing, 28(1), 4047. 